(C) 2007 The Japan Society of Applied Physics

\title{
New Design Concept and Fabrication Process for Three-Dimensional Silicon Photonic Crystal Structures
}

\author{
Daihei HIPPO ${ }^{1,3 *}$, Kei UraKawA ${ }^{2}$, Yoshiyuki Kawata ${ }^{1}$, Yoshishige TsuchiYA ${ }^{1,3}$, \\ Hiroshi MizUTA $^{1,3}$, Nobuyoshi KoshidA ${ }^{2,3}$, and Shunri OdA ${ }^{1,3}$ \\ ${ }^{1}$ Department of Physical Electronics and Quantum Nanoelectronics Research Center, Tokyo Institute of Technology, \\ 2-12-1 O-okayama, Meguro-ku, Tokyo 152-8552, Japan \\ ${ }^{2}$ Graduate School of Engineering, Tokyo University of Agriculture and Technology, 2-24-16 Naka-cho, Koganei, Tokyo 184-8588, Japan \\ ${ }^{3}$ SORST JST (Japan Science and Technology Agency), 4-1-8 Honcho, Kawaguchi, Saitama 332-0012, Japan
}

(Received July 20, 2006; revised September 27, 2006; accepted November 13, 2006; published online February 8, 2007)

We propose a new design concept and a fabrication process for three-dimensional (3D) silicon photonic crystals on a $100 \mathrm{~nm}$ scale that does not require any alignment processes. The elemental technique used in this process is two directional electrochemical etching processes at a particular magnetic field. First, we have performed photonic band calculation and estimated device parameters to obtain the maximum photonic band gap in the visible range centered at around $800 \mathrm{~nm}$. Next, we have experimentally observed the formation of a two-dimensional periodic pore with a diameter of $80 \mathrm{~nm}$ and an aspect ratio above 80 on an $\mathrm{n}^{+}$(100) silicon substrate. Finally, we have fabricated 3D microstructures by two directional etching processes. A clear directionality for the pore formation was observed in two directions, showing the possibility of projecting the patterns formed on the slope to the side of the wafer. These fundamental etching processes can be applied to the fabrication of 3D photonic crystals in the visible range without any alignment processes. [DOI: 10.1143/JJAP.46.633]

KEYWORDS: 3D photonic crystal, photonic band gap, nanostructure fabrication, anodization, two directional etching processes

\section{Introduction}

Recently, considerable effort has been invested in the fabrication of three-dimensional (3D) photonic crystals. ${ }^{1-4)}$ In particular, downscaling and increasing the number of layers are critical to obtaining the highest performance for individual fabrication processes. However, most of the processes suffer from the difficulties in alignment between layers when the number of layers is increased, particularly in the small spectral range centered at around $1 \mu \mathrm{m}$. In addition, increasing the number of layers itself is a hard task, if ordinary dry etching processes are applied to realize layerby-layer structures.

On the other hand, a relatively simple fabrication process called an anisotropic wet process can also be applied to realize woodpile structures by two directional etching processes. The merits of the anisotropic wet process are mainly based on deep etching properties with an aspect ratio above 100, and the number of layers can be increased simply by changing the number of initial patterns on the side. Recently, the fabrication of a 3D silicon photonic crystal with a large photonic band gap in the near infrared spectral range of $1.25-1.66 \mu \mathrm{m}$ has been reported. ${ }^{1)}$ Photochemical etching and subsequent focused-ion-beam (FIB) drilling have been adopted. However, there has also been a misalignment of approximately $65 \mathrm{~nm}$ from the theoretically optimized value between the two processes, and the measured reflectivity has not exceeded $60 \%$ in this spectral range.

In the following section, we present a new fabrication process for full-band-gap inverse woodpile structures with a pore diameter below $200 \mathrm{~nm}$, involving two directional etching processes based on anodic oxidation at a magnetic field. The great merit of this method is the possibility of fabricating a full-band-gap woodpile structure in a number of layers at once in an extremely small spectral range centered at around $800 \mathrm{~nm}$ and no additional alignment

*E-mail address: d-hippo@neo.pe.titech.ac.jp processes are required. Furthermore, the fabrication of a new type of structure, such as an inverse woodpile structure, would be possible, which has the same photonic band gap properties as woodpile structures. The inverse woodpile structure is more structurally stable and has more diverse physical properties, such as its applicability to multi wavelength light sources which are discussed in the next section.

\section{Theory and Design of 3D Photonic Crystal Structures}

The original concept of the inverse woodpile structure was proposed by several researchers around 1994, even though they did not necessarily use this term explicitly. ${ }^{5,6)}$ In this study, photonic band calculations are carried out on the basis of the structure proposed by Fan et al., as shown in Fig. $1 .{ }^{5)}$ First, we briefly describe the principle of the plane wave expansion method used in these calculations.

Starting from the Maxwell curl eqs. (1) and (2), we assume that all materials are linear, lossless and isotropic, so that $D=\varepsilon_{0} \varepsilon(x) E$ and $B=\mu_{0} H$, where the dielectric function $\varepsilon(x)$ is the square of the refractive index.

$$
\begin{aligned}
& \nabla \times E(x, t)=-\frac{\partial B}{\partial t} \\
& \nabla \times H(x, t)=\frac{\partial D}{\partial t}
\end{aligned}
$$

Taking time-independent solutions $E(x)=E(x) \exp (-i \omega t)$ and $H(x)=H(x) \exp (-i \omega t)$, we can combine the Maxwell equations to obtain the vector Helmholtz eq. (3).

$$
\nabla \times\left(\frac{1}{\varepsilon(x)} \nabla \times\right) H(x)=\frac{\omega^{2}}{c^{2}} H(x)
$$

Using Bloch's theorem, we write $H(x)=\exp (i k \cdot x) u_{k}(x)$, where $u_{k}(x)$ is a function with a lattice periodicity. Inserting this expression in the Helmholtz eq. (3), we obtain

$$
\hat{L} u_{k}=(i k+\nabla) \times\left(\frac{1}{\varepsilon(x)}(i k+\nabla)\right) \times u_{k}=\bar{\omega}^{2} u_{k},
$$




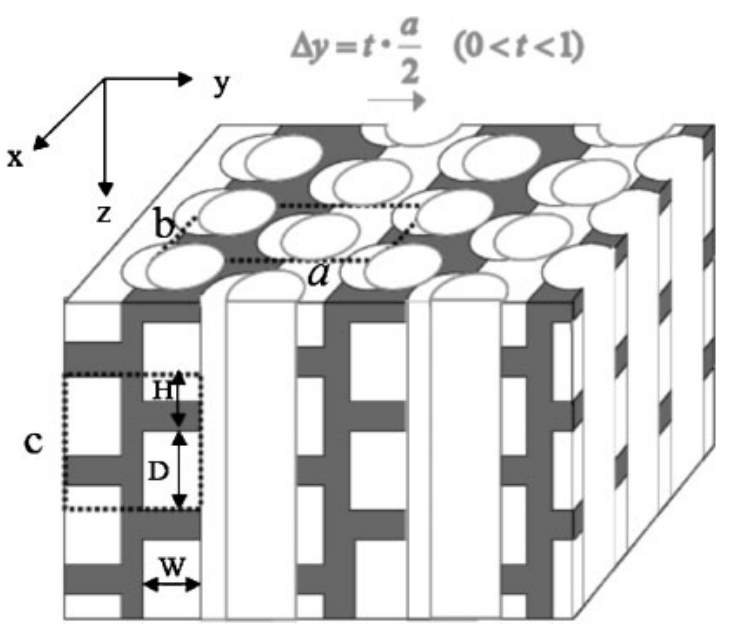

(a)

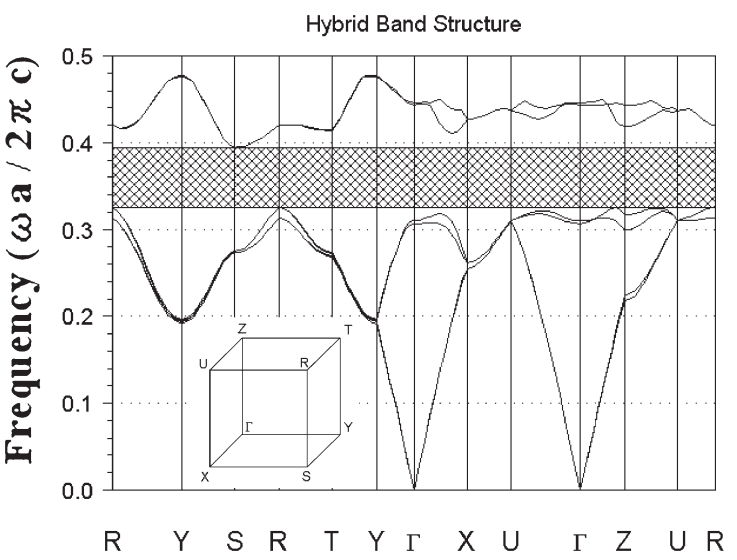

(b)

Fig. 1. (a) 3D photonic crystal structure proposed by Fan et al. ${ }^{5)}$ and parameters used for photonic band calculation. White regions indicate air holes, whereas other regions consist of silicon. (b) The plane wave expansion method is used to calculate the photonic band structure. A normalized photonic band gap of $19.3 \%$ is obtained from the calculation. The parameters used in this calculation are $W=0.36 a, D=0.38 a, R=$ $0.22 a, b=0.71 a$, and $H=0.28 a$, where $a$ is the period of the photonic crystal in the $y$-direction [see (a)]. The inset shows the irreducible Brillouin zone of the simple orthorhombic lattice used in this calculation. 5 )

where $\hat{L}$ is the operator and $\bar{\omega}=\omega / c$ is the normalized frequency.

Solving this eigenvalue eq. (4) with the wavevector $k$ as a free parameter, we obtain the photonic band structures.

Note that this method can treat only lossless materials, although silicon is supposed to absorb photons in the visible range of interest. We should calculate the photonic band structures by the finite difference time domain (FDTD) method to treat this problem precisely. However, in this paper, we neglect the absorption effect in the first-order approximation to analyze the systematic changes in the photonic band gap properties, without considering the substrate material, actual device scale or sample surface condition. In actual cases, we should note two points concerning the absorption effect. First, the absorption edges of the mesoporous and microporous silicon are blue shifted compared with that of bulk silicon and show a low absorption coefficient in the visible range. ${ }^{7)}$ Second, although the free carriers in heavily doped silicon absorb

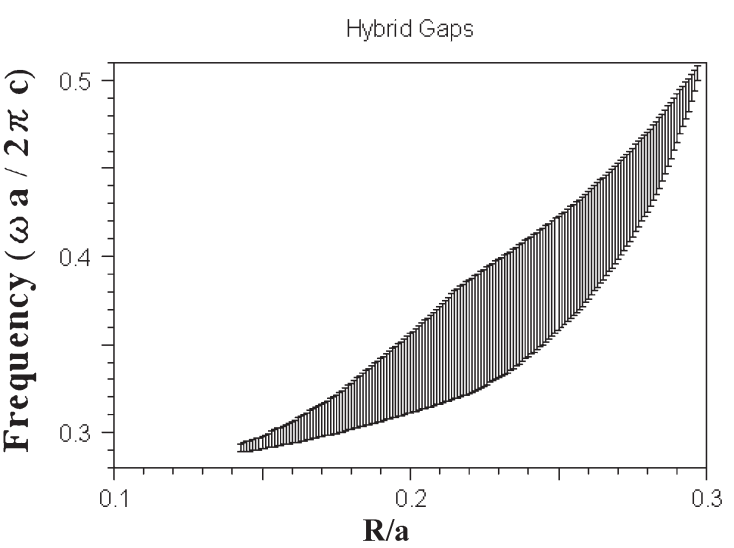

(a)

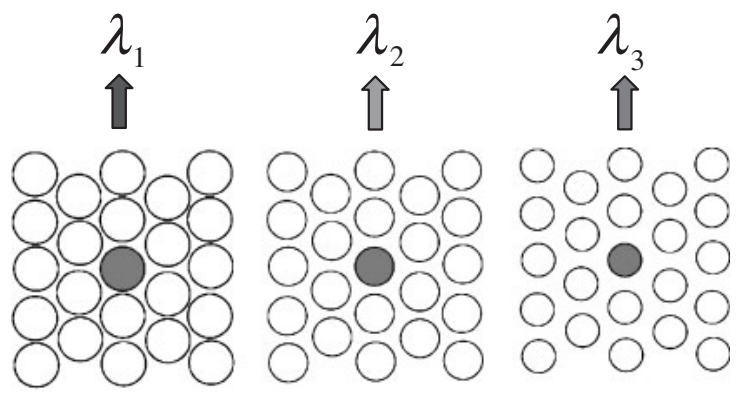

(b)

Fig. 2. (a) Calculated photonic band gap map. The horizontal axis represents the pore diameter on the top surface divided by the period of the photonic crystal in the $y$-direction [see Fig. 1(a)]. The central frequency of the photonic band gap increases with the pore diameter on the top surface. (b) A schematic of the multiwavelength light source coupled with 3D photonic crystals with a single defect. The figure shows the center plane of the whole 3D structure. The black circle in the center of each pattern indicates the position of the light emitter. The wavelength of the emitted light is increased by decreasing the pore diameter on the top surface.

photons below the fundamental band gap, ${ }^{8)}$ if such substrates are made mesoporous by anodization, the absorption coefficient of the dry layers decreases by orders of magnitude with increasing porosity. ${ }^{9)}$ The decreased absorption coefficient for the infrared regime in mesoporous silicon has been interpreted as being caused by the trapping of free charge carriers at deep levels related to the surface states. ${ }^{10}$ These two effects are enhanced by increasing the porosity, in which 3D photonic crystals are expected to have the most prominent features.

According to the above-mentioned calculations, this structure has an excellent property with a normalized photonic band gap of about $20 \%$. This structure can be fabricated by two directional etching processes on the silicon substrate; the first directional etching is performed from the top, and the second directional etching, from the side. Thus, the number of layers can be simply controlled by changing the initial pattern on the top surface. In addition, if we change the pore diameter on the top surface, we can control the central wavelength of the photonic band gap, as shown in Fig. 2(a). Figure 2(b) illustrates the multiwavelength light source coupled with 3D photonic crystals with a single defect. The figure shows the center plane of the whole 3D structure. The black circle in the center of each pattern 


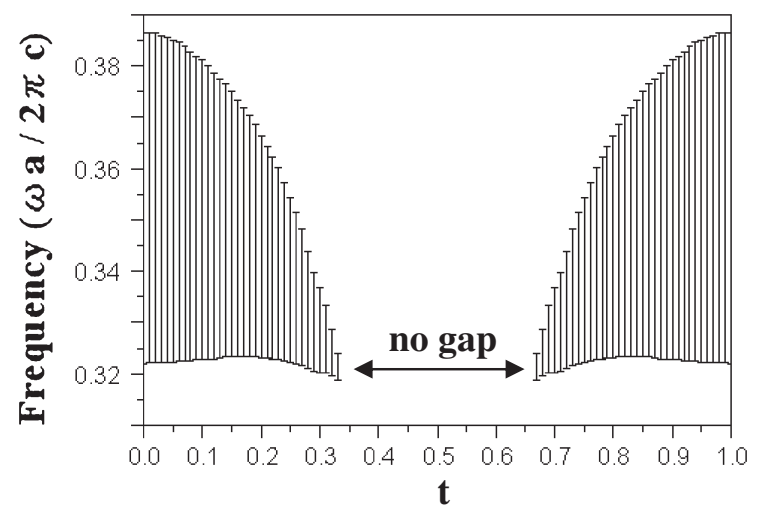

Fig. 3. Calculated photonic band gap map. The horizontal axis $t$ represents the ratio of misalignment between two directional etching processes [see Fig. 1(a)]. The parameters $t=0$ and $t=1$ correspond to the same photonic crystal structure. This figure shows that the misalignment of about $30 \%$ of a half period in the $y$-direction inhibits the formation of the photonic band gap.

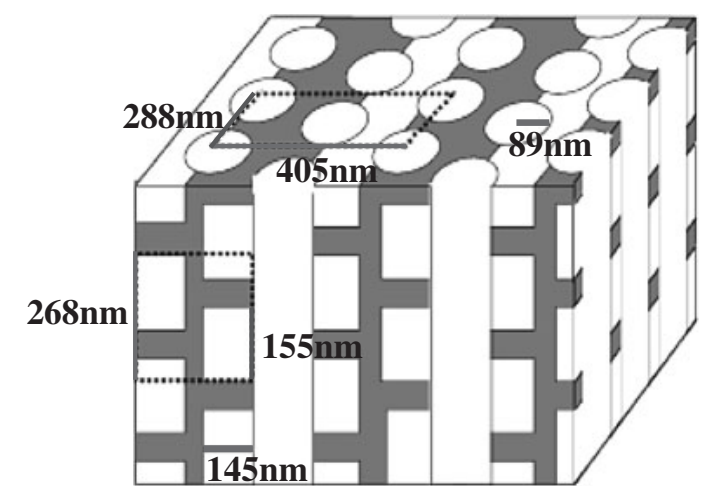

Fig. 5. Device dimensions corresponding to central wavelength of photonic band gap at $800 \mathrm{~nm}$. The calculated pore diameter is less than $200 \mathrm{~nm}$.

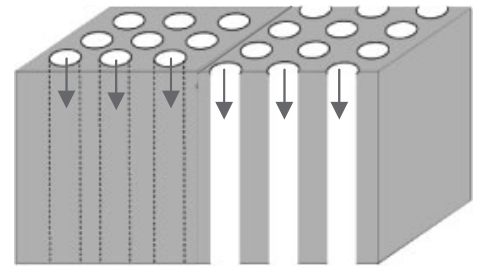

(a)

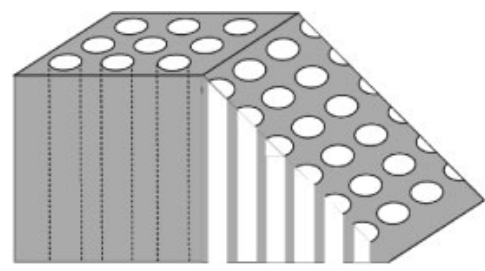

(b)

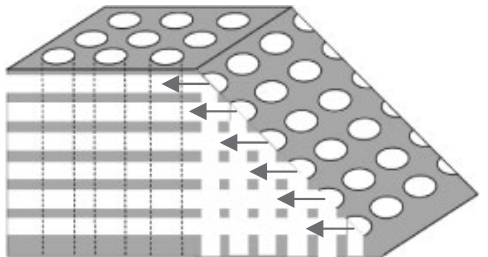

(c)

Fig. 4. Fabrication processes of inverse woodpile structure. (a) First directional etching, (b) mechanical polishing, and (c) second directional etching.

indicates the position of the light emitter. The wavelength of the emitted light is increased by decreasing the pore diameter on the top surface. As a result, we can simply obtain multiwavelength light sources by changing the initial pattern on the top surface. This is one of the great merits of the inverse woodpile structure.

As for the fabrication process, the most important factor in generating a large photonic band gap is the alignment between two directional etching processes. To estimate the margin of the misalignment between two directional etching processes to generate a photonic band gap, we have calculated the dependence of the photonic band gap on the positional displacement of the two processes, as shown in Fig. 3. On the basis of the results of this calculation, the misalignment of about $30 \%$ of a half period in the $y$ direction [indicated in Fig. 1(a)] is sufficient for inhibiting the formation of the photonic band gap. The gap disappears when two directional etching processes have the same phase, whereas the gap is maximized when two directional etching processes are exactly half-period-shifted. Therefore, in the first process, we propose the fabrication of two types of pattern on the top surface only half-period-shifted at a straight boundary, as shown in Fig. 4. In the second process, the slope is formed along the straight boundary by mechanical polishing. At this stage, the patterning of pores on the slope is realized. At the final stage, the second directional etching is performed in the direction perpendicular to the first directional etching using the pattern formed on the slope as a guide.

The device dimensions obtained for the 3D full photonic band gap (20\%) at $800 \mathrm{~nm}$ are shown in Fig. 5. As we can observe in this figure, both directional etchings require the formation of a pore with a diameter below $200 \mathrm{~nm}$. We have adopted magnetic field-assisted anodization instead of photoelectrochemical etching for which only pores with diameters larger than $400 \mathrm{~nm}$ have been reported. ${ }^{1)}$ The mechanism of magnetic field-assisted anodization relies on the control of the motion of holes that are responsible for initiating the electrochemical dissolution of silicon. ${ }^{11)}$ The directional etchings are enhanced by the preferential supply of holes with a velocity component parallel to the applied magnetic field.

\section{Experimental Results and Discussion}

The samples were prepared using heavily doped (0.04 $\Omega \cdot \mathrm{cm})$ n-type $\mathrm{Si}(100)$ wafers. Anodization was performed in the dark in dilute $\mathrm{HF}(10 \%)$ solution at a constant current density of $12 \mathrm{~mA} / \mathrm{cm}^{2}$ for $10 \mathrm{~min}$. The temperature of the solution was kept at $0{ }^{\circ} \mathrm{C}$ during the anodization. The first directional etching was performed at an external magnetic field applied to the $\mathrm{Si}$ substrate perpendicular to the surface. The magnitude of the applied magnetic field was $1.9 \mathrm{~T}$.

The result of the first directional etching is shown in Fig. 6. Figure 6(a) shows the straight vertical etching with a pore diameter of $80 \mathrm{~nm}$ and an aspect ratio above 80 realized 


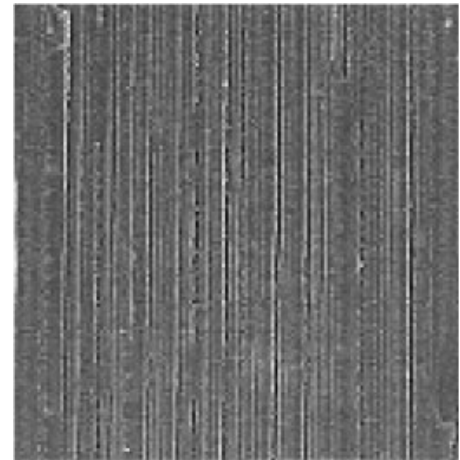

$1.5 \mu \mathrm{m}$

(a)

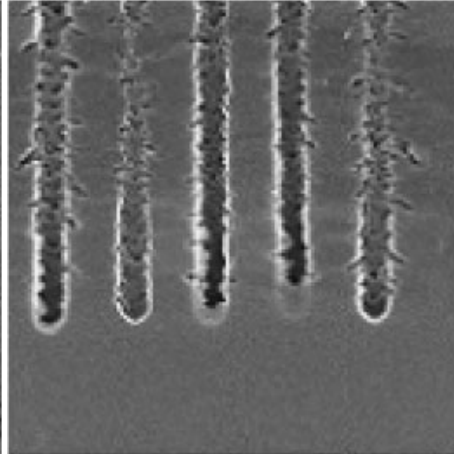

$0.2 \mu \mathrm{m}$

(b)

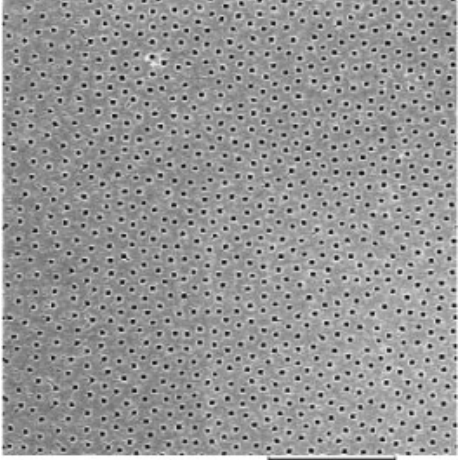

$2.5 \mu \mathrm{m}$

(c)

Fig. 6. Straight vertical etching with pore diameter of $80 \mathrm{~nm}$ and aspect ratio above 80 realized by anodization at magnetic field. Crosssectional SEM images of (a) middle (b) bottom parts of pores, and (c) SEM image of mechanically polished surface at a depth of $10 \mu \mathrm{m}$.

using this method. Figure 6(b) shows the bottom of the pores. Next the sample surface was mechanically polished by about $10 \mu \mathrm{m}$ to determine how the initial pattern on the top surface was projected in the deep region. Figure 6(c) shows the surface scanning electron microscope (SEM) image at a depth of $10 \mu \mathrm{m}$. As can be observed from this image, the geometry of the formed pores is in good agreement with the initial pattern on the top surface indicated in Fig. 5, although a slight deviation from the designed parameter cannot be avoided.

However, the most essential problem that is peculiar to all the two directional etching processes is that it is difficult to protect the existing pores during the second directional etching performed by either electrochemical etching or dry etching. In this case, the pore walls cannot be protected from further dissolution during the second directional etching when the pores are filled with HF, as shown in Fig. 7(a). Filling the pores with other materials is considered to be the best method of protecting the pore walls; among these

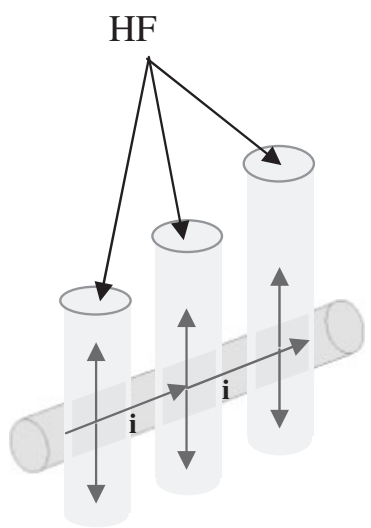

(a)

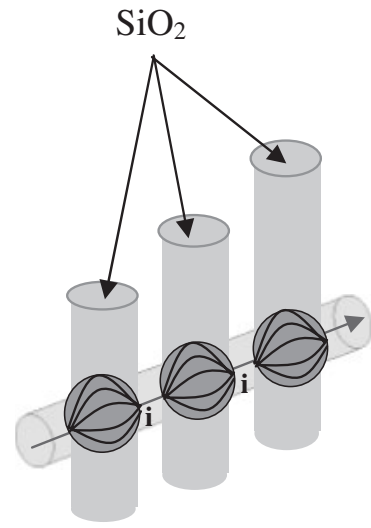

(b)
Fig. 7. (a) The pores on the slope formed in the first directional etching are filled with HF. In this case, the pore walls cannot be protected from further dissolution during the second directional etching. (b) The pores are filled with silicon dioxide. In this case, the dissolution proceeds along the right arrow indicated in the figure. It consists of two processes: one is the dissolution of silicon by electrochemical etching, and the other, the dissolution of silicon dioxide by isotropic etching in HF. materials, silicon dioxide is the most appropriate material for this purpose. There are many reasons for choosing this material. First, the dissolution rate of silicon dioxide in HF is much lower than that of silicon; thus, the pore walls are protected from HF immersion during the second directional etching. Second, silicon dioxide has a higher potential than silicon for holes that reduces the surface recombination rate on the existing pore walls during the second directional etching. When the pores are filled with silicon dioxide, the second directional etching is considered to proceed along the right arrow indicated in Fig. 7(b). It consists of two different processes. One is the dissolution of silicon by electrochemical etching and the other is the dissolution of silicon dioxide by isotropic etching in HF. The second directional etching is realized by repeating both dissolution processes.

Thus, the sample was thermally oxidized for $1 \mathrm{~h}$ at $1100{ }^{\circ} \mathrm{C}$ after the first directional etching to fill the pores with silicon dioxide, as shown in Fig. 8(a). Next the slope was formed by mechanical polishing, as shown in Fig. 8(b). Finally, the second directional etching was performed in the direction perpendicular to the first directional etching according to the pattern formed on the slope. The result is shown in Fig. 8(c). The directions of individual etching processes are indicated by arrows in the figure. We can clearly observe the directionality of each pore, although the pore diameters cannot be controlled well in the present state. To control both the diameters and directions of the pores, we need to optimize the etching conditions so that we can control the pore positions precisely in the first directional etching.

\section{Conclusions}

In conclusion, we proposed a new fabrication process for 3D silicon photonic crystals on a $100 \mathrm{~nm}$ scale. First, we performed photonic band calculation and estimated device parameters to obtain the maximum photonic band gap in the visible range centered at $800 \mathrm{~nm}$. Then, we showed the applicability of two directional etching processes to the fabrication of multiwavelength light sources. However, the estimated margin of the alignment error between two directional etching processes was so small that the misalign- 


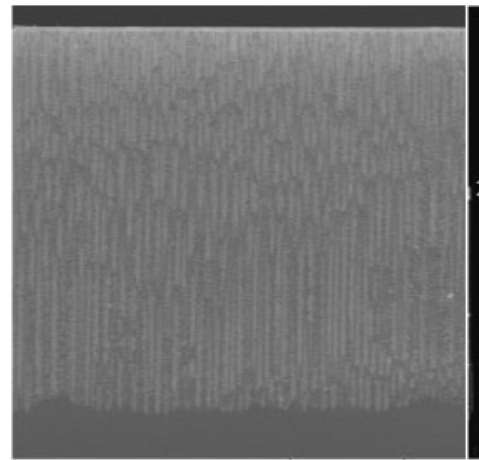

$5.5 \mu \mathrm{m}$

(a)

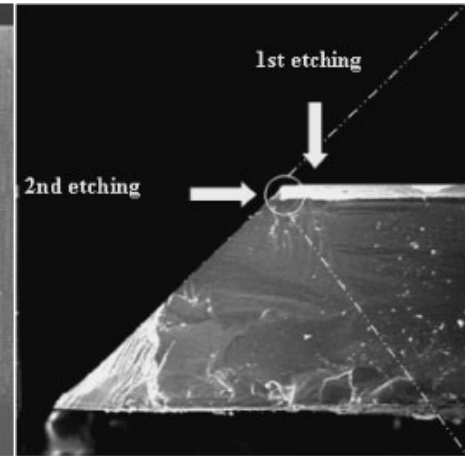

$250 \mu \mathrm{m}$

(b)

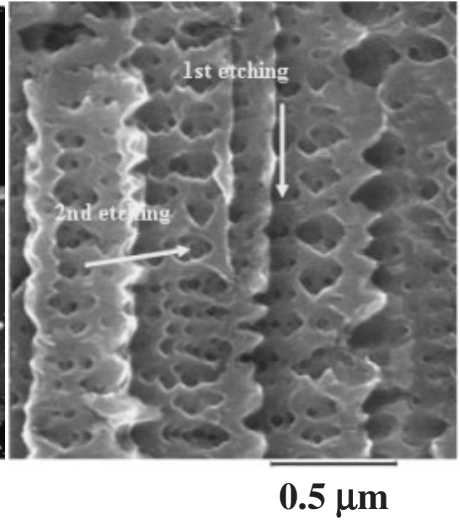

(c)

Fig. 8. (a) Cross-sectional SEM images of oxidized pores, (b) slope formed on sample, and (c) partially formed 3D microstructures on $100 \mathrm{~nm}$ scale after two directional etching processes.

ment of about $30 \%$ of a half period in one direction inhibits the formation of the photonic band gap. To circumvent this problem, we proposed a new fabrication process for $3 \mathrm{D}$ silicon photonic crystals that does not require any alignment processes. The key elemental technique is two directional electrochemical etching processes at a magnetic field, utilizing the pattern formed on the slope. We observed the formation of two-dimensional periodic pores with diameters of $80 \mathrm{~nm}$ and aspect ratios above 80 on the $\mathrm{n}^{+}(100)$ silicon substrate. The formed pores reflect well the initial pattern on the top surface, although a slight deviation from the designed parameter cannot be avoided. Next we fabricated the 3D silicon microstructures by two directional etching processes. We observed a clear directionality of the pore formation in two directions, showing the possibility of projecting the slope pattern on the side of the wafer. These fundamental etching processes can be applied to the fabrication of the $3 \mathrm{D}$ photonic crystals in the visible range without any alignment processes.
1) J. Schilling, J. White, A. Scherer, G. Stupian, R. Hillebrand, and U. Gösele: Appl. Phys. Lett. 86 (2005) 011101.

2) S. Noda, K. Tomoda, N. Yamamoto, and A. Chutinan: Science 289 (2000) 604.

3) M. Qi, E. Lidorikis, P. T. Rakish, S. G. Johnson, J. D. Joannopoulos, E. P. Ippen, and H. I. Smith: Nature 429 (2004) 538.

4) S. Matthias, F. Müller, C. Jamois, R. B. Wehrspohn, and U. Gösele: Adv. Mater. 16 (2004) 2166.

5) S. Fan, P. R. Villeneuve, R. D. Meade, and J. D. Joannopoulos: Appl. Phys. Lett. 65 (1994) 1466.

6) K. M. Ho, C. T. Chan, C. M. Soukoulis, R. Biswas, and M. Sigalas: Solid State Commun. 89 (1994) 413.

7) D. Kovalev, G. Polisski, M. Ben-Chorin, J. Diener, and F. Koch: J. Appl. Phys. 80 (1996) 5978.

8) R. A. Soref and B. R. Bennett: IEEE J. Quantum Electron. 23 (1997) 123.

9) M. H. Chan, S. K. So, and K. W. Cheah: J. Appl. Phys. 79 (1996) 3273.

10) V. Lehmann, F. Hofmann, F. Möller, and U. Grüning: Thin Solid Films 255 (1995) 20.

11) T. Nakagawa, H. Sugiyama, and N. Koshida: Jpn. J. Appl. Phys. 37 (1998) 7186. 